\title{
Caracterización De Los Consumidores De Ropa De Moda
}

\author{
Dra. Adriana Méndez Wong \\ Mtra. Edith Reyes Ruiz. \\ Dr. Jesús Francisco Mellado Siller \\ Karina Lizbeth Rodriguez Ramirez. \\ Universidad Autónoma de Coahuila, Mexico
}

Doi:10.19044/esj.2018.v14n31p148 URL:http://dx.doi.org/10.19044/esj.2018.v14n31p148

\begin{abstract}
This study is a Marketing work about the life style of the consumers related to their fashion clothing purchases. For that an instrument is applied using a scale proposed by Chegedzai, Manillall and Lawrence Mandhalazi (2014) who identified seven constructs for the typology: Fashion Aware, Hedonist, Brand Aware, Innovative, Quality Aware, Undecisive and Brand Loyalty. Its a transversal, quantitative, exploratory and descriptive investigation. The sample shows 1000 individuals from 21 to 80 years old, in Saltillo, Coahuila, Mexico, validating a 4o items instrument acquiring an Alfa de Cronbach of 0.952. The questions were categorical and the answers were indicted on a Likert Scale from 1 to 5. The results on relation to the analyzed dimensions, point that the interviewed attempt to buy quality clothes and aknowledge that it is an effort to achieve it because their incomes are a limiting to their purchase of High End clothing brands. The genre affects on the purchase decision, concluding that there are meaningful differences between men and women: the women enjoy more buying clothes, they have more fun while they're doing it and dedicate more time to the activity mentioned. It is a Project from the Red Nacional de Investigación "Gestion de la Mercadotecnia" del Consorcio de Universidades Mexicanas. (National Net of Investigation "Marketing management" of the Mexican Universities Consortium).
\end{abstract}

Keywords: Consumer Behavior, Consumer Investigation, Life Style

\section{Resumen}

El estudio es un trabajo del Marketing acerca del estilo de vida de los consumidores relacionado con sus compras de ropa de moda. Para ello se aplica un instrumento utilizando la escala propuesta por Chengedzai, Manillall y Lawrence Mandhlazi (2014), quienes identificaron siete constructos para la 
tipología: Consciente de la moda, Hedonista, Consciente de la marca, Innovador, Consciente de la Calidad, Indecisos y Lealtad a la Marca. Es una investigación de corte transversal, cuantitativo, exploratorio y descriptivo. La muestra es de 1000 sujetos de 21 a 80 años de edad, en la ciudad de Saltillo Coahuila, México, validando un instrumento de 40 ítems obteniendo un Alfa de Cronbach de 0.952. Las preguntas fueron categóricas y las respuestas se indicaron en escala de Likert de 1 a 5 . Los resultados en relación a las dimensiones analizadas, señalan que los entrevistados procuran comprar ropa de calidad y reconocen que es un esfuerzo conseguirlo pues sus ingresos son una limitante para la adquisicion de ropa de marcas prestigiadas. El género sí influye en las decisiones de compra, concluyendo que existen diferencias significativas en los hombres y mujeres: las mujeres disfrutan más el comprar la ropa, se divierten mientras lo hacen y dedican más tiempo a dicha actividad. Es un proyecto de la Red Nacional de Investigación "Gestión de la Mercadotecnia" del Consorcio de Universidades Mexicanas.

Palabras Claves: Comportamiento del Consumidor, Investigación del Consumidor, Estilos de vida.

\section{Introducción:}

En primer lugar, comprenderemos el objetivo del análisis del comportamiento del Consumidor como lo define Solar Pujals (2001), al mencionar que es explicar el por qué del comportamiento de éste, revelando los procesos racionales o emocionales de la compra y el porqué de la aceptación o rechazo de un producto. Las líneas de estudio son: motivacion de compra; quién decide la compra, quién influye, quién compra; actitudes cognitivas y emotivas; hábitos de compra, uso y consumo; estilo de vida, también denominados psicográficos o AIO (actitudes, intereses y opiniones); segmentación y tipología de los consumidores. La investigación del comportamiento del consumidor no mide al consumidor, mide sus actitudes, creencias, percepciones y preferencias.

\section{Desarrollo:}

Schiffman \& Lazar Kanuk (2010) señalan que el comportamiento del consumidor se enfoca en la forma en que los individuos toman decisiones para gastar sus recursos disponibles (tiempo, dinero y esfuerzo) en artículos relacionados con el consumo. Eso incluye lo que compran, por qué lo compran, cuándo lo compran, dónde lo compran, con qué frecuencia lo compran, cuán a menudo lo usan, cómo lo evalúan después y cuál es la influencia de tal evaluación en compras futuras, y cómo lo desechan. Así mismo comprender las influencias personales y grupales que afectan las decisiones de compra y la manera en que las toman. 
En una sociedad de consumo moderna las personas son más libres de seleccionar un conjunto de productos, servicios y actividades que los definen $\mathrm{y}$, a la vez, de crear una identidad social que comunican a los demás. La elección de bienes y servicios realmente afirma quienes somos y el tipo de personas con quien nos deseamos identificar, e incluso a aquellas que deseamos evitar. Una perspectiva de marketing de estilos de vida reconoce que las personas se separan en grupos con base en las cosas que les gustan, en la forma en que pasan su tiempo libre y en la manera en que deciden gastar sus ingresos. El estilo de vida es un patrón de consumo que refleja las decisiones de la gente sobre la forma en que gasta su tiempo y su dinero. (Blackwell, Miniard, \& Engel, 2002)

La escala Likert es el formato más popular para las escalas de actitudes, porque facilita a los investigadores la elaboración e interpretación, y a los consumidores les resulta fácil de responder. Ellos marcan o escriben el número correspondiente a su nivel de "acuerdo" o de "desacuerdo" con cada una de las declaraciones, en una serie que describe la actitud objeto de la investigación. La escala contiene el mismo número de opciones de acuerdo/desacuerdo a cada lado de la opción neutral. Una de las principales ventajas de la escala Likert es que brinda al investigador la posibilidad de considerar por separado las respuestas a cada declaración, o de combinar las respuestas para obtener una calificación general. ( Schiffman \& Lazar Kanuk, 2010)

En Estados Unidos en el año 2004, se realizó una investigación para analizar la conciencia de la moda entre los chinos, japoneses y los adolescentes estadounidenses (Parker, Hermans, \& Schaefer, 2004). El propósito del estudio fue examinar las similitudes y diferencias en las actitudes hacia la moda a través de estos tres mercados. Los resultados muestran que existen diferencias significativas en el sentido de la moda entre adolescentes chinos y sus homólogos japoneses y nosotros. Al mismo tiempo, los adolescentes norteamericanos y japoneses muestran similitudes en sus actitudes hacia la moda. Los resultados pueden apoyar la idea de las diferencias de mercado en el sentido de la moda entre los países desarrollados y los países menos desarrollados. Si bien hay oportunidades para los comerciantes de moda aprovechar las similitudes del mercado, una comprensión de la idiosincrasia subyace en las motivaciones de moda adolescente en cada mercado.

Chengedzai, Manillall, \& Lawrence (2014), realizan una investigación donde su objetivo principal fue examinar las tipologías de compradores de la Generación Y, considerando las variables edad y moda de ropa; utilizaron como instrumento una encuesta y su muestra fue de 230 sujetos pertenecientes a la Generación Y. Realizando el Análisis factorial exploratorio para identificar los tipos de compradores, aplicaron la prueba de Kruskal-Wallis para examinar la influencia de la edad sobre las tipologías de compradores 
identificados. El estudio identificó siete tipologías de compradores que son aplicables a los millenials.

\section{METODOLOGÍA}

Tabla 1. Diseño del Estudio

\begin{tabular}{|cc|}
\hline Tipo de investigación & $\begin{array}{c}\text { Cuantitativa, descriptiva y transversal no } \\
\text { experimental }\end{array}$ \\
Tamaño de la Muestra & 1,000 sujetos \\
Procedimiento de Muestreo & Aleatorio simple \\
Error muestral & $4.0 \% ; \mathrm{p}=\mathrm{q}=0.5 ;$ nivel de confianza $95 \%$ \\
Método de trabajo de campo & $\begin{array}{c}\text { Encuesta auto administrada } \\
\text { Cuestionario }\end{array}$ \\
Cuestionario estructurado de 40 ítems, \\
utilizando la escala desarrollada por \\
Chengedzai, Manillall y Lawrence \\
compuesto de siete variables. Las preguntas \\
son categóricas y la escala de respuesta es tipo \\
Likert del 1 al 5. Incluyéndose 9 reactivos de \\
tipo sociodemográfico. \\
Saltillo, Coahuila, México.
\end{tabular}

Fuente: Elaboración propia.

Tabla 2. Resultados de las pruebas de fiabilidad.

\begin{tabular}{rr}
\hline \hline Alfa de Cronbach & N de elementos \\
\hline .952 & 40 \\
\hline \hline
\end{tabular}

Fuente: Elaboración propia

Ítems de la escala:

1. Consciente de la moda: Característica representativa de un consumidor quien está motivado a mantenerse al día con tendencia de moda y estilos

2. Hedonista: Característica para medir el grado en donde un consumidor encuentra la actividad de comprar disfrutable y compra solo por diversión.

3. Consciente de la marca: Mide la orientación de un consumidor para lo más caro y de marcas reconocidas.

4. Innovador: Identifica consumidores que gustan de productos nuevos e innovadores y se emocionan buscando nuevos productos. 
5. Consciente de la calidad: Característica que mide el grado en el que un consumidor busca cuidadosa y sistemáticamente la mejor calidad en productos.

6. Indecisos: Identifica al consumidor que percibe muchas marcas y tiendas de donde escoger, así como información.

7. Lealtad a la marca: Indica al consumidor que tiene marcas y tiendas favoritas y que han formado hábitos cerca de ellas.

Los ítems del Constructo Consciente de la Moda son:

1. La ropa a la moda significa mucho para mí

2. Soy un experto usando ropa a la moda

3. La ropa a la moda es una parte significativa en mi vida

4. Usualmente visto de moda

5. Estoy interesado en la ropa de moda

6. Voy de compras para mantener las tendencias de moda

7. Me es familiar la ropa a la moda

8. Siento que conozco todo acerca de la ropa a la moda

9. Me considero un experto en moda

10. Para mí la ropa a la moda es un producto importante

Ítems del Constructo Hedonista:

1. Comprar ropa no es una actividad placentera

2. Ir a comprar ropa es una de las actividades que más disfruto en mi vida

3. Disfruto comprar por diversión

4. Hago mis compras rápidamente

5. No pierdo tiempo solo comprando

6. Comprar ropa es tiempo perdido

7. Sí vale mi tiempo al comprar en las tiendas

8. Comprar ropa satisface mi sentido de curiosidad

Los ítems del Constructo Consciente de la Marca:

1. Mayor costo en la ropa, mejor calidad

2. Tiendas departamentales agradables y de especialidad me ofrecen la mejor ropa

3. Las marcas que más se anuncian, usualmente son las mejores opciones

4. Las marcas de ropa reconocidas son las mejores para mí

5. Las marcas de ropa más caras usualmente se compran por decisión

6. Prefiero comprar marca de ropa más vendidas

Los ítems del Constructo Innovador:

1. Mantengo mi guardarropa actualizado con la moda cambiante

2. El estilo fashionista y atractivo es muy importante para mí

3. Para tener variedad, compro en tiendas diferentes y escojo marcas diferentes

4. Es divertido comprar ropa nueva 
Los ítems del Constructo Consciente de la Calidad:

1. Conseguir muy buena calidad es importante para mí

2. Cuando de comprar ropa se trata, trato de conseguir lo mejor o tomar la decisión perfecta

3. En general, trato de comprar la mejor de la calidad en ropa

4. Hago un esfuerzo especial para escoger la mejor calidad en ropa

5. Mis estándares y expectativas en la ropa que compro son muy altos Los ítems del Constructo Indecisos:

1. Hay muchas marcas de donde escoger y frecuentemente me siento confundido

2. A veces, es difícil escoger en que tienda comprar

3. Mientras más aprendo de ropa, más difícil me es escoger la mejor

4. Toda la información que tengo en diferentes productos me confunde Los ítems del Constructo Lealtad a la marca:

1. Tengo marcas favoritas que compro una y otra vez

2. Una vez que encuentro una marca que me gusta, me apego a ella

3. Voy a las mismas tiendas cada vez que compro ropa

Considerando la información que se recolectó en este trabajo de investigación y las variables de segmentación que fueron establecidas en el instrumento de recolección de datos, se consideró pertinente analizar la información obtenida mediante una prueba de análisis de la varianza de un factor (ANOVA), mediante la que se analizaron, de manera sistemática, las medias de la muestra para determinar si los datos provienen de la misma población objeto de estudio, y determinar, de esta manera, el nivel de significancia existente entre las variables analizadas y sus respectivos factores de segmentación.

\section{Resultados}

El perfil demográfico de los encuestados es que sus edades oscilan entre 21 y 80 años; el $40.7 \%$ de los entrevistados son hombres y el $59.3 \%$ son mujeres; respecto a su ocupación el $37.4 \%$ es estudiante, $23.9 \%$ empleado, $11.3 \%$ profesionista independiente, $22.8 \%$ hogar y $4.6 \%$ desempleado; en relación con el estado civil el $44.1 \%$ manifiesta ser soltero, un $42.8 \%$ casado, otros un $9 \%$ y un $4.1 \%$ divorciado; en cuanto a nivel de estudios, un $5.2 \%$ con nivel de posgrado, un $40.2 \%$ con Licenciatura, un $29.8 \%$ con bachillerato, 9.3\% de Primaria y $1.1 \%$ no cuentan con preparación académica.

El análisis requerido para la información de este estudio fué el de la varianza de un factor (ANOVA) con el que será posible comprobar si existen diferencias significativas entre cada una de los cuarenta ítems o preguntas que integran el constructo consumidor de ropa de moda. Por lo tanto, los resultados obtenidos del perfil de los consumidores procedentes de Saltillo Coahuila se presentan en las tablas que se muestran a continuación: 
Tabla 2. Situación media de los consumidores de prendas de vestir a la moda ${ }^{1}$

\begin{tabular}{|c|c|c|}
\hline Variables & Medias & Significancia \\
\hline Soy un experto usando ropa a la moda & 1.77 & \\
\hline Siento que conozco todo acerca de la ropa a la moda & 1.88 & ** \\
\hline Me es familiar la ropa a la moda & 2.12 & \\
\hline Voy de compras para mantener las tendencias de moda & 2.02 & \\
\hline Voy a las mismas tiendas cada vez que compro ropa & 2.72 & \\
\hline $\begin{array}{c}\text { Una vez que encuentro una marca que me gusta, me apego a } \\
\text { ella }\end{array}$ & 2.65 & \\
\hline La ropa a la moda es una parte significativa en mi vida & 1.99 & \\
\hline Me considero un experto en moda & 1.94 & \\
\hline A veces, es difícil escoger en que tienda comprar & 2.65 & \\
\hline Comprar ropa es tiempo perdido & 2.54 & $* * *$ \\
\hline No pierdo tiempo solo comprando & 2.92 & $* * *$ \\
\hline Hago mis compras rápidamente & 2.92 & $* * *$ \\
\hline Disfruto comprar por diversión & 2.42 & $* * *$ \\
\hline Cuando de comprar ropa se trata, trato de conseguir lo mejor & 3.00 & $* * *$ \\
\hline Conseguir muy buena calidad es importante para mi & 2.91 & $* * *$ \\
\hline Es divertido comprar ropa nueva & 2.95 & $* * *$ \\
\hline Para tener variedad, compro en tiendas diferentes & 2.73 & $* * *$ \\
\hline Tengo marcas favoritas que compro una y otra vez & 2.67 & ** \\
\hline Toda la información que tengo en diferentes productos & 2.30 & \\
\hline Mientras más aprendo de ropa, más difícil me es escoger & 2.26 & \\
\hline Las marcas de ropa reconocidas son las mejores para mi & 2.34 & \\
\hline Las marcas que más se anuncian & 2.40 & \\
\hline Tiendas departamentales agradables & 2.59 & \\
\hline Mayor costo en la ropa, mejor calidad & 2.51 & \\
\hline Comprar ropa satisface mi sentido de curiosidad & 2.28 & $* *$ \\
\hline Si vale mi tiempo al comprar en las tiendas & 2.49 & $* * *$ \\
\hline El estilo fashionista y atractivo es muy importante para mi & 2.00 & \\
\hline Mantengo mi guardarropa actualizado & 2.13 & \\
\hline Prefiero comprar marca de ropa más vendidas & 2.34 & \\
\hline Las marcas de ropa más caras & 2.91 & \\
\hline Ir a comprar ropa es una de las actividades que más disfruto & 2.48 & $* * *$ \\
\hline Comprar ropa no es una actividad placentera & 2.64 & $*$ \\
\hline Para mí la ropa a la moda es un producto importante & 2.17 & \\
\hline Hay muchas marcas de donde escoger & 2.31 & \\
\hline Mis estándares y expectativas en la ropa que compro & 2.46 & \\
\hline Hago un esfuerzo especial para escoger la mejor calidad & 2.96 & \\
\hline En general, trato de comprar la mejor de la calidad en ropa & 2.89 & $*$ \\
\hline Estoy interesado en la ropa de moda & 2.47 & \\
\hline Usualmente visto de moda & 2.44 & \\
\hline $\begin{array}{l}\text { La ropa a la moda significa mucho para mi } \\
\qquad \mathrm{N} \text { válido (según lista) }\end{array}$ & 2.28 & \\
\hline
\end{tabular}

${ }^{1}$ En una escala de $1=$ total desacuerdo a $5=$ total acuerdo

Diferencias estadísticamente significativas: $(*): \mathrm{p}<0.1 ;(* *): \mathrm{p}<0.05 ;(* * *): \mathrm{p}<0.01$.

Fuente: Elaboración propia 
La tabla 2 muestra que las tres principales variables en orden de importancia para los Consumidores de Ropa son: Cuando de comprar ropa se trata, trato de conseguir lo mejor, con una media de 3.00 en una escala de $1=$ total desacuerdo a $5=$ total acuerdo como límites, seguida de la variable Hago un esfuerzo especial para escoger la mejor calidad con una media de 2.96 y, finalmente, Es divertido comprar ropa nueva con una media de 2.95.

Además se observa que son diez las variables en las que existen diferencias estadísticamente significativas: Comprar ropa es tiempo perdido , No pierdo tiempo solo comprando, Hago mis compras rápidamente, Disfruto comprar por diversión, Cuando de comprar ropa se trata, trato de conseguir lo mejor, Conseguir muy buena calidad es importante para mí, Es divertido comprar ropa nueva, Para tener variedad, compro en tiendas diferentes, Si vale mi tiempo al comprar en las tiendas e Ir a comprar ropa es una de las actividades que más disfruto.

\section{Constructo Consumidor Hedonista}

Referente al Constructo Consumidor Hedonista, que es la característica para medir el grado en donde un consumidor encuentra la actividad de comprar disfrutable y compra sólo por diversión, al aplicar el estadístico Prueba de Kruskal-Wallis nos encontramos que 5 de 8 ítems tiene un nivel de significancia $<0.05$, lo que representa que si existen diferencias significativas entre hombres y mujeres (Tabla 3).

Tabla 3: Prueba de Kruskal-Wallis del Constructo Consumidor Hedonista

ÍTEMS

$\begin{array}{ll}\text { CHI- } & \text { SIG. } \\ \text { CUADRADO } & \text { ASINTÓT. }\end{array}$

\begin{tabular}{llll}
\hline COMPRAR ROPA NO ES UNA ACTIVIDAD & 4.373 & .037 \\
PLACENTERA & 5.582 & .018 \\
IR A COMPRAR ROPA ES UNA DE LAS & & \\
ACTIVIDADES QUE MÁS DISFRUTO EN MI & 7.923 & .005 \\
VIDA & 3.834 & .050 \\
DISFRUTO COMPRAR POR DIVERSIÓN & .159 & .690 \\
HAGO MIS COMPRAS RÁPIDAMENTE & .052 & .819 \\
NO PIERDO TIEMPO SOLO COMPRANDO & 4.881 & .027 \\
COMPRAR ROPA ES TIEMPO PERDIDO & 1.246 & .264 \\
SI VALE MI TIEMPO AL COMPRAR EN LAS & & \\
TIENDAS & & \\
COMPRAR ROPA SATISFACE MI SENTIDO DE & & \\
CURIOSIDAD & & \\
\hline
\end{tabular}

Prueba de Kruskal-Wallis $*_{p}<.05$ Variable de agrupación: Género. Esta tabla muestra que del grupo de 8 items que integran el Constructo Hedonista, cinco de ellos tiene una significancia < a 0.05, por lo que se puede afirmar con una confianza al 95\% que si hay diferencias significativas entre hombres y mujeres con respecto a ser consumidores hedonistas.

Fuente: Elaboración propia 
Tabla 4. Tabulación cruzada Género y Comprar ropa no es una actividad placentera

\begin{tabular}{|c|c|c|c|c|c|c|c|c|}
\hline & & & \multicolumn{5}{|c|}{ Comprar ropa no es una actividad placentera } & \multirow[t]{2}{*}{ Total } \\
\hline & & & $\begin{array}{l}\text { Totalmente } \\
\text { Desacuerdo }\end{array}$ & Desacuerdo & Indeciso & Acuerdo & $\begin{array}{l}\text { Totalmente } \\
\text { De Acuerdo }\end{array}$ & \\
\hline \multirow{6}{*}{ Genero } & & Recuento & 43 & 27 & 40 & 13 & 12 & 135 \\
\hline & HOMBRE & $\begin{array}{l}\% \text { del } \\
\text { total }\end{array}$ & $16.5 \%$ & $10.3 \%$ & $15.3 \%$ & $5.0 \%$ & $4.6 \%$ & $51.7 \%$ \\
\hline & \multirow[b]{2}{*}{ MUJER } & Recuento & 37 & 12 & 36 & 21 & 20 & 126 \\
\hline & & $\begin{array}{c}\% \text { del } \\
\text { total }\end{array}$ & $14.2 \%$ & $4.6 \%$ & $13.8 \%$ & $8.0 \%$ & $7.7 \%$ & $48.3 \%$ \\
\hline & \multirow[b]{2}{*}{ Total } & Recuento & 80 & 39 & 76 & 34 & 32 & 261 \\
\hline & & $\begin{array}{c}\% \text { del } \\
\text { total }\end{array}$ & $30.7 \%$ & $14.9 \%$ & $29.1 \%$ & $13.0 \%$ & $12.3 \%$ & $100.0 \%$ \\
\hline
\end{tabular}

Fuente: Elaboración propia

En la tabla 4, al afirmar que el comprar ropa no es una actividad placentera, el $45.6 \%$ está en desacuerdo con la declaración, el $29.1 \%$ es indeciso y sólo el $25.3 \%$ el comprar ropa no les da placer.

Tabla 5 Tabulación cruzada Género y Disfruto comprar por diversión

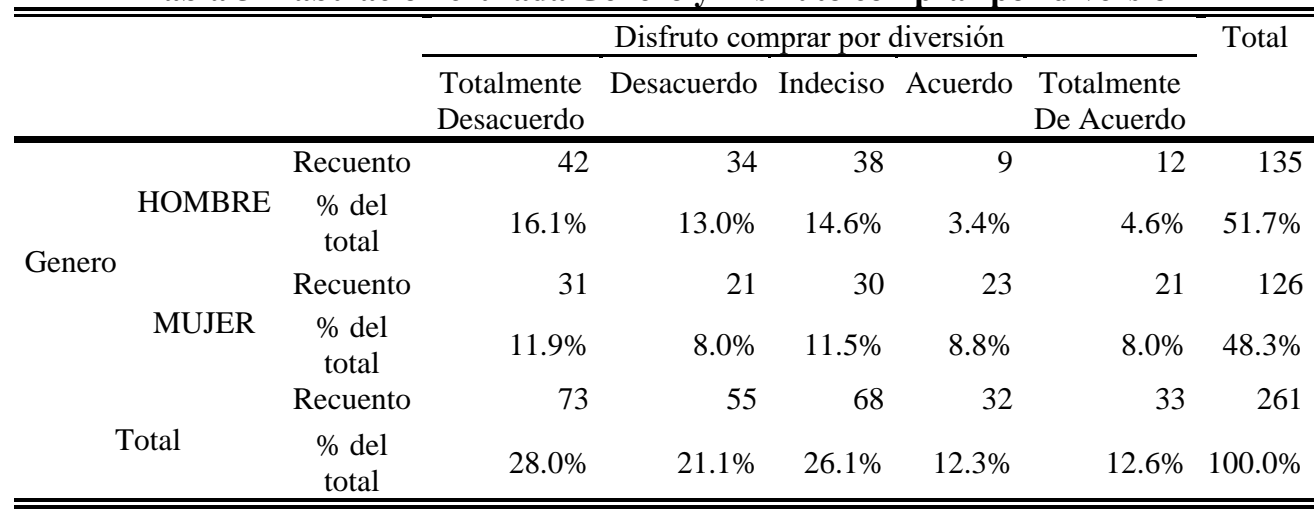

Fuente: Elaboración propia

En la tabla 5 se manifiesta al preguntar si disfruta comprar por diversión, en donde el $24.9 \%$ afirman divertirse cuando lo hacen, siendo las mujeres quien más lo disfrutan con un $16.8 \%$.

Por lo tanto se encuentran resultados que muestran que existen diferencias significativas en los hombres y mujeres, ya que el género femenino considera que las compras son placenteras.

\section{Conclusion:}

Los resultados con base en las variables analizadas, nos muestran que el género si afecta y determina sus decisiones de compra de prendas de vestir, especialmente en los consumidores hedonistas. Se reconoce el hecho de que las compras son acordes a los estilos de vida, teniendo una misma opinión con Blackwell, Miniard \& Engel (2002), de que a menudo elegimos un producto 
precisamente porque está asociado con cierto estilo de vida, que en los saltillenses está latente la necesidad de reconocimiento y aceptación social. Se puede confirmar empíricamente que no tan fácilmente se guían por la moda para decidir sus compras de ropa. Concluyendo que según los resultados si existen diferencias significativas en los hombres y mujeres: las mujeres disfrutan más el comprar la ropa, se divierten mientras lo hacen y dedican más tiempo a dicha actividad. Asimismo, se detecta la limitante de los ingresos y el nivel socioeconómico al que pertenecen los entrevistados, para la adquicisión de ropa de marcas de prestigio.

\section{References:}

1. Blackwell, R. D., Miniard, P. W., \& Engel, J. F. (2002). Comportamiento del Consumidor. Mexico: International Thomson Editores.

2. Chengedzai , M., Manillall , D., \& Lawrence, M. (2 de Abril de 2014). 'Shopper typologies amongst a Generation $Y$ consumercohort and variations in terms of age in the fashionapparel market'. Recuperado el 26 de Enero de 2015, de http://www.actacommercii.co.za: http://dx.doi.org/10.4102/ac.v14i1.209

3. Mafini, C., Dhurup, M., \& Mandhlazi, L. (2014). Shopper typologies amongst a Generation $\mathrm{Y}$ consumer cohort and variations in terms of age in the fashion apparel market. Acta Commercii.

4. Parker, R. S., Hermans, C. M., \& Schaefer, A. D. (2004). "Fashion consciousness of Chinese, Japanese and American teenagers". Journal of Fashion Marketing and Management: An International Journal, 176-186.

5. Schiffman, L. G., \& Lazar Kanuk, L. (2010). Comportamiento del Consumidor (DÉCIMA EDICIÓN ed.). México: PEARSON EDUCACIÓN.

6. Soler Pujals, P. (2001). Investigación de mercados. Barcelona, España: Universidad Autónoma de Barcelona. 